OPEN ACCESS

Edited by: Yang Deng,

Qingdao Agricultural University, China

Reviewed by:

Guoliang Yan,

China Agricultural University, China

Madhumita Barooah,

Assam Agricultural University, India

*Correspondence:

Wei Su

wsu@gzu.edu.cn

Specialty section:

This article was submitted to

Food Microbiology,

a section of the journal

Frontiers in Microbiology

Received: 31 December 2019

Accepted: 18 March 2020

Published: 17 April 2020

Citation:

Jiang L, Su W, Mu Y and Mu Y

(2020) Major Metabolites

and Microbial Community of Fermented Black Glutinous Rice

Wine With Different Starters.

Front. Microbiol. 11:593.

doi: 10.3389/fmicb.2020.00593

\section{Major Metabolites and Microbial Community of Fermented Black Glutinous Rice Wine With Different Starters}

\author{
Li Jiang ${ }^{1,2}$, Wei Su ${ }^{1,2 *}$, Yingchun $M u^{1,2}$ and $Y u u^{1}$ \\ ${ }^{1}$ School of Liquor and Food Engineering, Guizhou University, Guiyang, China, ${ }^{2}$ Guizhou Key Laboratory for Fermentation \\ Engineering and Biopharmaceuticals, Guizhou University, Guiyang, China
}

Black glutinous rice wine (BGRW) is a traditional Chinese rice wine that is brewed using multiple strains. However, the roles of these microorganisms, particularly their contributions to aroma formation, are poorly understood. Accordingly, the main goal of this study was to determine the microbial communities and major metabolites of different traditional fermentation starters. Anshun (AS) starter and Xingyi (XY) starter were used for BGRW to provide insight into their potential contributions to the variation in flavor and aroma. High-throughput sequencing of the microbial community using the Illumina MiSeq platform revealed significant differences during fermentation between the two starter groups. Pediococcus, Leuconostoc, and Bacillus were the dominant bacterial genera in the AS group, whereas Leuconostoc, Pediococcus, and Gluconobacter were the dominant genera in the $X Y$ group. In addition, Rhizopus, Saccharomyces, and Saccharomycopsis were the predominant fungal genera detected in both samples. The major metabolites in the two groups were identified by high-performance liquid chromatography and headspace-solid-phase microextraction gas chromatographymass spectrometry. A total of seven organic acids along with 47 (AS) and 43 (XY) volatile metabolites were detected, among which lactic acid was the primary organic acid, and esters were the largest group in both types of wine. Principal components analysis further revealed significant differences in the dynamic succession of metabolites between the two samples. Correlation analysis showed that 22 and 17 microorganisms were strongly correlated with the production of major metabolites in AS and XY, respectively. Among them, Pediococcus, Leuconostoc, Lactobacillus, Lactococcus, and Streptococcus were shown to play crucial roles in metabolite synthesis. Overall, this study can provide a valuable resource for the further development and utilization of starters to improve the aromatic quality of BGRW.

Keywords: black glutinous rice wine, microbial diversity, high-throughput sequencing, flavor, correlation 


\section{INTRODUCTION}

Guizhou black glutinous rice wine (BGRW) is a traditional Chinese rice wine that is distinguished by the use of the local specialty black glutinous rice as the raw material, conferring unique characteristics to flavors and tastes. Black glutinous rice (Oryza sativa $L$ ), as one of the most popular varieties of brown rice, has been called "black pearl," owing to its high content of phenols, flavonoids, and especially anthocyanins (Shen et al., 2009). Anthocyanins are antioxidants with nutritive properties that can help suppress the rate of cell damage and thus prevent carcinogenesis (Hou, 2003; Fimognari et al., 2008). Hence, consumption of black rice or black rice products can increase celldefensive actions and prevent reactive oxygen species-induced diseases (Junka et al., 2016). Black glutinous rice wine is very popular in the Chinese market because the nutrients in the raw materials are dissolved in the wine, resulting in a product that is enriched in protein, amino acids, and multivitamins (Zhao, 2016). In addition, BGRW has many potential medicinal properties, including antioxidant ability and a protective effect on the kidney (Su et al., 2015; Qi et al., 2018). Thus, the use of black glutinous rice as a raw material not only can provide a variety of rice wines that are enriched in nutrients with benefits for physiological activities but also can meet the increasing consumer demands for healthier products.

The traditional BGRW brewing process involves inoculation of a starter in the glutinous rice, saccharification in an open environment, and subsequent aging for fermentation. The Anshun (AS) starter and Xingyi (XY) starter in the Guizhou province were fermented by Rhizopus inoculated from rice meal and herbs, with relatively high saccharification and liquefaction capabilities, and high wine yield, making them the first choice for traditional BGRW fermentation. For AS starter, white peony (Paeonia suffruticosa Andr., Paeoniaceae), hizoma chuanxiong (Ligusticum chuanxiong Hort., umbelliferae), and asarum (Asarum sieboldii Miq., Aristolochiaceae) were used. For $\mathrm{XY}$ starter, white peony ( $P$. suffruticosa Andr., Paeoniaceae), mint (Mentha haplocalyx Briq., Labiatae), mulberry leaf (Morus alba L., Moraceae), and common vladimiria root (Aucklandia lappa Decne., Compositae) were used. The microbial species, along with their relative abundances and interactions, in the starter cultures play an important role in fermentation, which determines the ultimate productivity and flavor quality of the rice wine (Hong et al., 2016; Yang et al., 2017a). For instance, mold and some bacteria can produce liquefaction and saccharification enzymes, which hydrolyze the starch in the raw material to produce the fermentable sugars that the yeast consume to produce alcohol as a by-product (Cai et al., 2018). In addition, mold, yeast, and bacteria produce a variety of enzymes required for cellular metabolism that generates diverse small molecules, which further contribute to the flavor and aroma of the final products (Yang et al., 2017a). Therefore, it is of great significance to analyze the microflora and their major metabolites, along with their relationships, as a basis for improving the flavor quality of BGRW. Indeed, extensive research has been conducted to unravel the composition and dynamics of the microbial communities associated with Chinese rice wine ( $\mathrm{Lv}$ et al., 2012;
Tang et al., 2019). Most of these studies have been based on traditional molecular methods such as culture-dependent methods and culture-independent polymerase chain reaction (PCR)-denaturing gradient gel electrophoresis techniques (Lv et al., 2015, 2017; Liu et al., 2017). However, these methods have certain limitations in comprehensively reflecting the actual microbial diversity in a sample due to their low flux and low sensitivity.

As an alternative approach, the continuous development of high-throughput sequencing (HTS) technology now enables more in-depth and precise evaluations of complex microflora with a reasonably low cost and in a relatively short period (Delgado et al., 2014; Lee et al., 2016) and has thus been widely applied for comprehensive analyses of microorganisms in various fermented foods, such as Shaoxing rice wine (Nie et al., 2015), Fagopyrum tataricum (buckwheat) wine (Ren et al., 2019), and Sake (Atsushi et al., 2018). High-throughput sequencing-based analysis of bacterial 16S rRNA genes and fungal ITS genes showed correlations between the final rice wine quality and the microbial composition of the starter. However, HTS has not been used to study the dynamics of the microbial community during BGRW fermentation. Although previous studies on BGRW have focused on the volatile metabolites present (Su et al., 2017, 2018), the effect of microorganisms on the formation of these metabolites during fermentation has not been evaluated to date.

Therefore, the aim of the present study was to perform a more in-depth and accurate evaluation of the complex microbial community dynamics during the brewing process of BGRW. In particular, we attempted to provide detailed insight of the microflora of BGRW fermented using different starters based on identification with HTS technology, along with their associated organic acids and volatile compounds determined by highperformance liquid chromatography (HPLC) and headspacesolid-phase microextraction (HS-SPME) combined with gas chromatography-mass spectrometry (GC-MS). Finally, the potential correlations between the metabolites and microbiota were uncovered through Pearson correlation analysis. These results can provide new fundamental insight into the complex dynamics of microbial communities during BRGW fermentation, along with offering a reference for the technical optimization of BGRW production.

\section{MATERIALS AND METHODS}

\section{Sample Preparation and Collection}

The starters (AS and XY) used in this study were obtained from the cities of AS and XY of Guizhou Province, China. Black glutinous rice wine was brewed in a well-known local winery following conventional procedures. In brief, 10-kg black glutinous rice was washed and soaked in water overnight at room temperature $\left(12-19^{\circ} \mathrm{C}\right)$ and then steamed at $100^{\circ} \mathrm{C}$ for $1 \mathrm{~h}$. After the steamed rice was cooled using cold water, it was mixed with the starters and transferred to wine jars $(80 \mathrm{~L})$. The amount of AS sample added was 5\%, whereas $2 \%$ XY starter was added, reflecting their respective optimal growth rates. At the end of saccharification (2 days), sterile water (30 L) was added 
for a 24 -day traditional fermentation step at $25-30^{\circ} \mathrm{C}$. Triplicate independent brewing was conducted for each starter.

At $0,2,4,6,11,17$, and 24 days during fermentation, $10 \mathrm{~g}$ of the fermented glutinous rice was collected at random under aseptic conditions for DNA extraction and HTS. In addition, $500 \mathrm{~mL}$ of the fermentation mash was collected at different brewing phases under aseptic conditions to identify the organic acids and volatile compounds with HPLC and HS-SPME-GCMS. All samples were stored at $-80^{\circ} \mathrm{C}$ until analysis.

\section{DNA Purification and PCR Amplification}

Total genomic DNAs were extracted from samples using the Power Soil DNA Isolation Kit (MO BIO Laboratories, Carlsbad, CA, United States) according to the manufacturer's protocol. DNA quality and quantity were assessed by the ratios of $260 / 280 \mathrm{~nm}$ and $260 / 230 \mathrm{~nm}$. Then, DNA was stored at $-80^{\circ} \mathrm{C}$ until further processing. The $\mathrm{V} 3-\mathrm{V} 4$ region of the bacteria $16 \mathrm{~S}$ rRNA gene was amplified with primers 338F (5'-ACTCCTACGGGAGGCAGCAG-3') and 806R (5'GGACTACHVGGGTWTCTAAT-3') (Zhang et al., 2015), and the ITS1 region of the fungi was amplified with the forward primer ITS1F (5'-CTTGGTCATTTAGAGGAAGTAA- $\left.3^{\prime}\right)$ and the reverse primer ITS1R (5'-GCTGCGTTCTTCATCGATGC3') (Buée et al., 2009).

Polymerase chain reaction amplification was carried out using as previously described (Miya et al., 2015). A total volume of $50 \mu \mathrm{L}$, which contained $10 \mu \mathrm{L}$ buffer, $0.2 \mu \mathrm{L}$ Q5 high-fidelity DNA polymerase, $10 \mu \mathrm{L}$ high GC enhancer, $1 \mu \mathrm{L}$ dNTP, $10 \mu \mathrm{M}$ of each primer, and $60 \mathrm{ng}$ genomic DNA. Thermal cycling conditions were as follows: an initial denaturation at $95^{\circ} \mathrm{C}$ for $5 \mathrm{~min}$, followed by 15 cycles at $95^{\circ} \mathrm{C}$ for $1 \mathrm{~min}, 50^{\circ} \mathrm{C}$ for $1 \mathrm{~min}$, and $72^{\circ} \mathrm{C}$ for $1 \mathrm{~min}$, with a final extension at $72^{\circ} \mathrm{C}$ for $7 \mathrm{~min}$. The PCR products from the first-step PCR were purified through VAHTSTM DNA Clean Beads (Vazyme Biotech Co., Ltd., Nanjing, China). A second-round PCR was then performed in a $40 \mu \mathrm{L}$ reaction, which contained $20 \mu \mathrm{L} 2 \times \mathrm{Ph} \mu$ sion HF MM, $8 \mu \mathrm{L}$ dd $\mathrm{H}_{2} \mathrm{O}, 10 \mu \mathrm{M}$ of each primer, and $10 \mu \mathrm{L}$ PCR products from the first step. Thermal cycling conditions were as follows: an initial denaturation at $98^{\circ} \mathrm{C}$ for $30 \mathrm{~s}$, followed by 10 cycles at $98^{\circ} \mathrm{C}$ for $10 \mathrm{~s}, 65^{\circ} \mathrm{C}$ for $30 \mathrm{~s}$ min, and $72^{\circ} \mathrm{C}$ for $30 \mathrm{~s}$, with a final extension at $72^{\circ} \mathrm{C}$ for $5 \mathrm{~min}$. Successful PCR amplification was verified by $1.8 \%$ agarose gel electrophoresis. All of PCR products were pooled, purified by gel extraction, and quantified using the AxyPrepDNA gel extraction kit (Axygen Corporation, Union City, CA, US) and the QuantiFluor ${ }^{\text {TM }}$-ST blue fluorescence quantitative system (Promega Corporation, Madison, Wisconsin, US). The purified PCR products were then mixed at equimolar ratios for sequencing on an Illumina HiSeq PE150 system (Illumina Corporation, San Diego, CA, United States) by Biomarker Technologies Co., Ltd (Beijing, China).

\section{Physiochemical Properties Determination}

Physical and chemical indexes such as total sugar, alcohol, total acid, and amino nitrogen were tested according to Chinese national standard (GB/T 13662, 2018).

\section{Determination of Flavor Compounds Organic Acids Analysis}

The analysis of organic acids in the samples was performed by HPLC, according to Yu (2014), with some modifications. A 5-mL sample in a tube was centrifuged at 10,000 revolutions/min for $10 \mathrm{~min}$ and filtrated through a $0.45-\mu \mathrm{m}$ microporous membrane. The separations were carried out using an Agilent 1260 Infinity II system (Agilent Technologies Inc., Palo Alto, CA, US), equipped with a $4.6 \times 150-\mathrm{mm}$ and a $5-\mu \mathrm{m}$ welch ultimate Zorbax SBAq column. The column temperature was set at $30^{\circ} \mathrm{C}$. A mixture of phosphate buffer $\left(0.02 \mathrm{~mol} / \mathrm{L} \mathrm{Na} \mathrm{Na}_{4}\right)$, adjusted with $5 \%$ (wt/vol) phosphate solution to $\mathrm{pH}$ (2.70), was used as the mobile phase. The flow rate was $1 \mathrm{~mL} / \mathrm{min}$ (1-4 $\mathrm{min}), 0.3 \mathrm{~mL} / \mathrm{min}$ (4-10 $\mathrm{min})$, and finally $1 \mathrm{~mL} / \mathrm{min}$ after $10 \mathrm{~min}$. The detection wavelength was $210 \mathrm{~nm}$.

\section{Volatile Compounds Analysis}

Volatile compounds in the BGRW samples were analyzed by HSSPME-GC-MS following the method described by Yang et al. (2017a), with modifications. Each sample $(5 \mathrm{~mL})$ was placed in a 20-mL SPME glass vial together with $1 \mathrm{~g}$ of sodium chloride and $10 \mu \mathrm{L}$ of the internal standard 2 -octanol $(40.34 \mathrm{mg} / \mathrm{L}$ in absolute ethanol). The fiber was headed into the SPME device, which was inserted in the vial and shaken at $50^{\circ} \mathrm{C}$ for $30 \mathrm{~min}$ to extract and absorb the volatile compounds. Then, it was desorbed in $7 \mathrm{~min}$ at $250^{\circ} \mathrm{C}$ into the GC inlet with the automatic autosampler. Volatiles analysis was carried out using a Trace GC Ultra gas chromatograph-DSQ II mass spectrometer (Thermo Electron Corp., Waltham, MA, United States) equipped with an DB-Wax column $(60 \mathrm{~m} \times 0.25 \mathrm{~mm} \times 0.25 \mathrm{~mm}$; Agilent Technologies $)$ and a flame ionization detector, attached to a mass spectrometer. The GC operation condition was as follows: an inlet temperature of $250^{\circ} \mathrm{C}$, split ratio of 10:1, and helium (purity: $99.999 \%$ ) carrier gas flow of $1 \mathrm{~mL} / \mathrm{min}$. The oven temperature program was as follows: $40^{\circ} \mathrm{C}(0 \mathrm{~min}), 4^{\circ} \mathrm{C} / \mathrm{min}$ to $150^{\circ} \mathrm{C}(5 \mathrm{~min}), 3^{\circ} \mathrm{C} / \mathrm{min}$ to $200^{\circ} \mathrm{C}$ $(6 \mathrm{~min})$, and finally $3^{\circ} \mathrm{C} / \mathrm{min}$ to $230^{\circ} \mathrm{C}(6 \mathrm{~min})$. The ion energy for the electron impact was kept at $70 \mathrm{eV}$. The chromatograms were recorded by monitoring the total ion currents in the 30350 mass range. The constituents were tentatively identified by matching the mass spectrum with the NIST5 spectrum database and verified by comparison of their Kováts retention indices (RIs) with the RI reported in literatures, calculated using C7-C40 n-alkanes.

\section{HTS and Sequence Analysis}

Raw sequencing data obtained from the Illumina platform were spliced using FLASH software (version 1.2.11) (Magoc and Salzberg, 2011) and filtered with Trimmomatic software (version 0.33). Finally, the UCHIME tool in QIIME software was used to remove any chimeras and retain only highquality tag sequences (Caporaso et al., 2010) (version 8.1). The UCLUST tool in QIIME software (version 1.8.0) was further used to cluster the tags at a $97 \%$ similarity level to obtain operational taxonomic units (OTUs), and taxonomic annotation was carried out on the obtained OTUs based on the Silva (bacteria) and UNITE (fungi) taxonomic databases (Edgar et al., 2011). The sample alpha-diversity 
indices were evaluated using MOTHUR software (version v.1.30). The relative abundances of the representative taxa were visualized with a columnar graph using EXCEL 2013 software (Microsoft Office, Redmond, Washington, US). Principal coordinate analysis (PCoA) was performed using QIIME software.

\section{Statistical Analysis}

Statistical analysis was performed using SPSS 17.0 (SPSS Inc., Chicago, IL, United States). The data obtained were subjected to one-way analysis of variance, and the significance level was considered at $P<0.05$. The organic acid data were mainly performed using Origin (version 2018) (Origin Lab Inc., Hampton, MS, United States). Principal component analysis (PCA) was performed to analyze the profiles of the volatile compounds using SIMCA software (version 14.1) (UMETRICS, Sweden). Pearson correlation was used to examine the correlation between microbial genera and major metabolites, and the correlation networks between the selected flavor metabolites and microbial community were visualized via the Cytoscape software (version 3.5.1). ${ }^{1}$ All experiments were completed in triplicates and data were expressed as means \pm standard deviations.

\section{RESULTS}

\section{Comparison of Microbial Community Diversity Among Samples}

The change in the microbial composition during BGRW fermentation with the two different starters was investigated by Illumina HiSeq sequencing analyses. The numbers of effective bacterial and fungi sequences were in the range of 66,411 to $70,493.33$ and $72,397.33$ to $77,216.67$ for AS, while 608,87 to $70,812.51$ and 72,608 to $76,985.67$ for XY, respectively (Supplementary Table S1). Fungal sequences greatly outnumbered those of bacteria in all samples; however, the total number of bacterial OTUs was far greater than that of fungi (Supplementary Table S1). In addition, the rarefaction curve based on OTUs approached the saturation plateau (Supplementary Figure S1), suggesting that the sequencing depth was adequate to represent the actual microbial structure of the samples. Dynamic changes to alpha-diversity indices during BGRW fermentation showed that richness and diversity of two samples were changed significantly during the fermentation process (Supplementary Table S2). Based on the 16S rRNA and ITS gene amplicons, Unifrac distance-based weighted PCoA was conducted to evaluate similarities and differences in the microbiota among the different samples. For the bacterial communities, clustered corresponding to the AS samples could be observed from 11 to 24 days of fermentation and to the XY samples could be observed from 6 to 24 days (Supplementary Figures S2A,B). For the fungal communities, clustered corresponding to the AS and XY samples could be observed from 2 to 24 days of fermentation (Supplementary Figures S2C,D).

\footnotetext{
${ }^{1}$ http://www.cytoscape.org/
}

\section{Microbial Community Dynamics During BGRW Fermentation}

Classification of 16S rRNA gene sequencing reads revealed 118 bacterial genera in the two samples. Microorganisms with a relative abundance greater than $1 \%$ were defined as core microorganisms, and their changes during fermentation are shown in Figure 1. With respect to the overall proportion, Pediococcus, Leuconostoc, and Bacillus were the dominant genera in AS, whereas Leuconostoc, Pediococcus, and Gluconobacter were the predominant genera detected in XY. At day 0, Pantoea (19.27\%) and Klebsiella (14.35\%) occupied the main position in AS samples, whereas Leuconostoc (12.40\%) was the predominant bacterial genus in the XY samples. From days 24 of fermentation, the microbial community of the AS samples was characterized by high ratios of Bacillus (37.10 and 67.10\%), whereas XY was characterized by Gluconobacter (40.34 and 18.75\%). From day 6 to 24, Pediococcus and Leuconostoc were the prevailing genera in AS. However, in XY, the proportion of Bacillus increased to the highest level of $64.09 \%$ at day 6 and then showed a subsequent rapid decline in parallel with the increase in abundance of Leuconostoc and Pediococcus.

The classification of ITS gene sequencing reads detected 52 fungal genera for the two samples. As shown in Figure 2, Rhizopus, Saccharomyces, Saccharomycopsis, and Aspergillus were identified as the core fungal genera in both wines. Nevertheless, some differences in the fungal communities were observed between AS and XY during the brewing process. The overall relative abundance of Rhizopus in AS was greater than that in XY, and this advantage was maintained until the end of fermentation. In XY, the relative abundance of Rhizopus dramatically increased

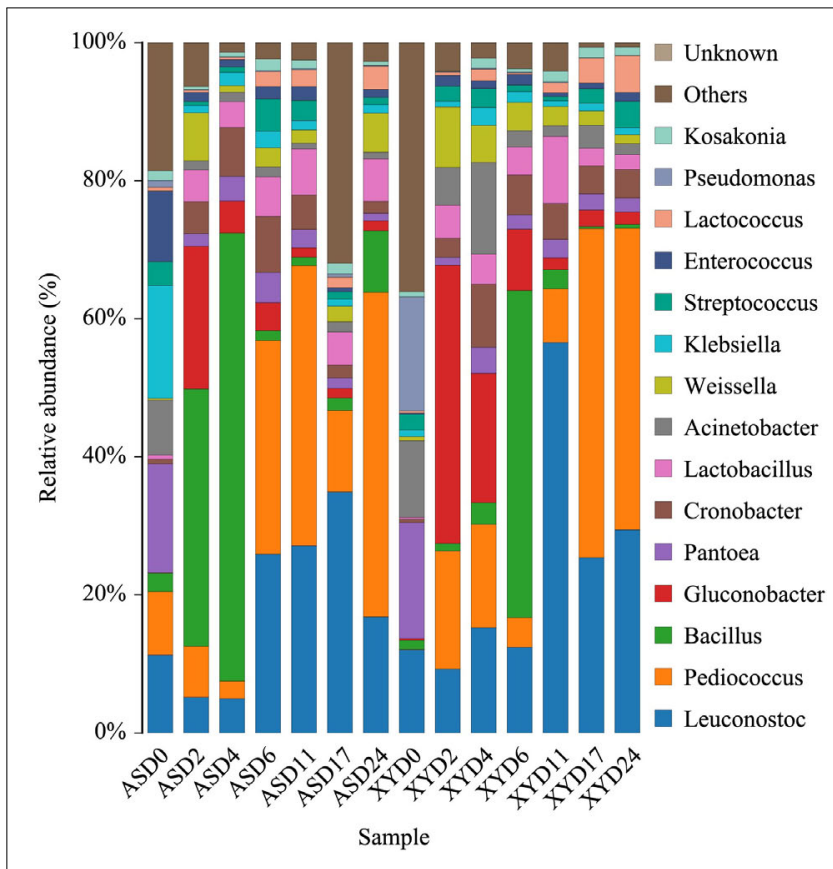

FIGURE 1 | Relative abundance of bacterial genera during different fermentation stages of black glutinous rice wine with two kinds of starters. 


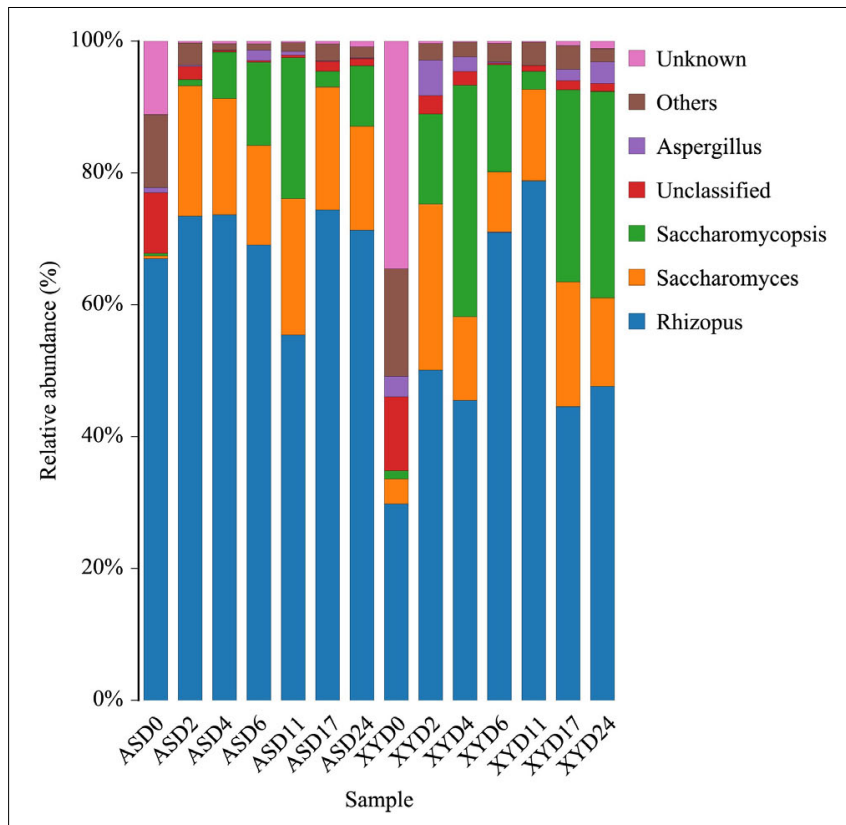

FIGURE 2 | Relative abundance of fungal genera during different fermentation stages of black glutinous rice wine with two kinds of starters.

from an initial value of 29.79 to $78.81 \%$ detected on day 11 of fermentation, followed by a subsequent rapid decrease to $46.61 \%$. However, the change in yeast strains (Saccharomyces, Saccharomycopsis) showed an opposite trend; thus, the increase in Saccharomyces and Saccharomycopsis was parallel to the decline in the abundance of Rhizopus in both starter groups. The overall proportion of Aspergillus in XY was higher than that in AS, with the highest proportion (0.05\%) detected at day 2 .

\section{Dynamics of Physicochemical Properties During BGRW Fermentation}

The physicochemical properties of the AS and XY samples during the different brewing stages (including total sugars, total acids, alcohol, and amino acid nitrogen) are summarized in Figure 3. Although the fermentation performance of the two samples showed similar trends during fermentation, differences between AS and XY were still observed. During fermentation, the total sugar concentration of the BGRW continuously diminished from an initial concentration of $51.34 \mathrm{~g} / \mathrm{L}$ to a final concentration of $19.72 \mathrm{~g} / \mathrm{L}$ in AS and from 48.41 to $18.48 \mathrm{~g} / \mathrm{L}$ in XY. Similarly, the alcohol content gradually increased from 5.07 to $18.42 \mathrm{vol} / \mathrm{vol} \%$ in AS and from 6.53 to $18.85 \mathrm{vol} / \mathrm{vol} \%$ in XY. According to the results of the microbial community summarized above, Rhizopus was dominant during the fermentation process, indicating that the glucoamylase produced by Rhizopus hydrolyzes starch into fermentable sugars for the yeast to convert into ethanol. Acids are immediately produced as fermentation proceeds. Indeed, the total acid content progressively increased during fermentation, and both samples showed the highest total acid content at the end fermentation, demonstrating that the acid-producing strains were most active in the later fermentation stage; however, XY
(8.39 g/L) showed a slightly higher final acid content than AS $(7.48 \mathrm{~g} / \mathrm{L})$. In addition, the content of amino acid nitrogen in the two wines showed an increasing trend, although in this case, the final acid content was greater in AS $(1.60 \mathrm{~g} / \mathrm{L})$ than in XY $(1.58 \mathrm{~g} / \mathrm{L})$ at the end of fermentation.

\section{Dynamics of Organic Acids and Volatile Compounds During BGRW Fermentation}

During fermentation, organic acids react with alcohols to generate esters, which are the main aroma compounds that can buffer and adjust the aroma and taste as well as improve the acidity of wine. Seven organic acids were detected in the two wines: pyruvate, lactic, oxalic, citric, succinic, malic, and tartaric acids. Figure 4 shows the trends of organic acids production during fermentation. Lactic acid was the primary acid detected, followed by malic acid. The lactic acid level in $\mathrm{XY}$ was significantly $(P<0.05)$ higher than that in AS, which showed a downward trend in both samples. However, the change of malic acid content in the two samples differed. The malic acid content in AS increased to the highest point at day 6 and then subsequently decreased until the end of fermentation. By contrast, in XY, the malic acid content declined at 4 and 24 days but showed an upward trend from 6 to 17 days. The content of oxalic acid and tartaric acid showed a decreasing trend in both samples, but the tartaric acid content in AS increased slightly at 17 days and then decreased thereafter. In addition, the levels of pyruvate and succinic acids both showed an upward trend. Compared with AS, the content of citric acid in XY fluctuated greatly, and the difference between the two samples was statistically significant $(P<0.05)$.

Forty-six volatile compounds were identified in AS, including 20 esters, 10 alcohols, 5 acids, 3 aldehydes, 3 phenols, and 5 others (alkanes, ketones, alkenes). Forty-three of these volatile compounds were detected in XY, including 20 esters, 9 alcohols, 3 acids, 3 aldehydes, 3 phenols, and 5 others (alkanes, ketones, alkenes). Although no significant $(P>0.05)$ differences in the type and amount of volatile compounds were observed between AS and XY, significant $(P<0.05)$ differences in the content of flavor compounds were detected (Figure 5). In general, the levels of alcohols (Figure 5A) and acids (Figure 5B) in AS were higher than those in XY, and some compounds, including benzyl alcohol (C9), isobutyric acid (S2), and butyric acid (S3), were found only in AS. After fermentation, both samples had higher concentrations of ethanol (C1), 2-methyl-1 propanol (C2), isoamyl alcohol (C4), and phenylethanol (C10). Overall, esters were the largest group detected in both types of wine (Figure 5C). Esters dominated the AS samples from day 2 to 17 of fermentation but were detected at significantly higher levels $(P<0.05)$ in the XY samples at day 24. At the end of the fermentation, AS was mainly characterized by ethyl lactate (Z3), octanoic acid ethyl ester (Z4), decanoic acid ethyl ester (Z7), and octanoic acid ethyl ester (Z4), whereas XY was mainly characterized by decanoic acid ethyl ester (Z8), phenyl ethyl acetate (Z13), myristic acid ethyl ester (Z16), ethyl 9-hexadecenoate (Z18), and linoleic acid ethyl ester (Z20). The aldehydes (Figure 5D) and phenols (Figure 5E) showed 


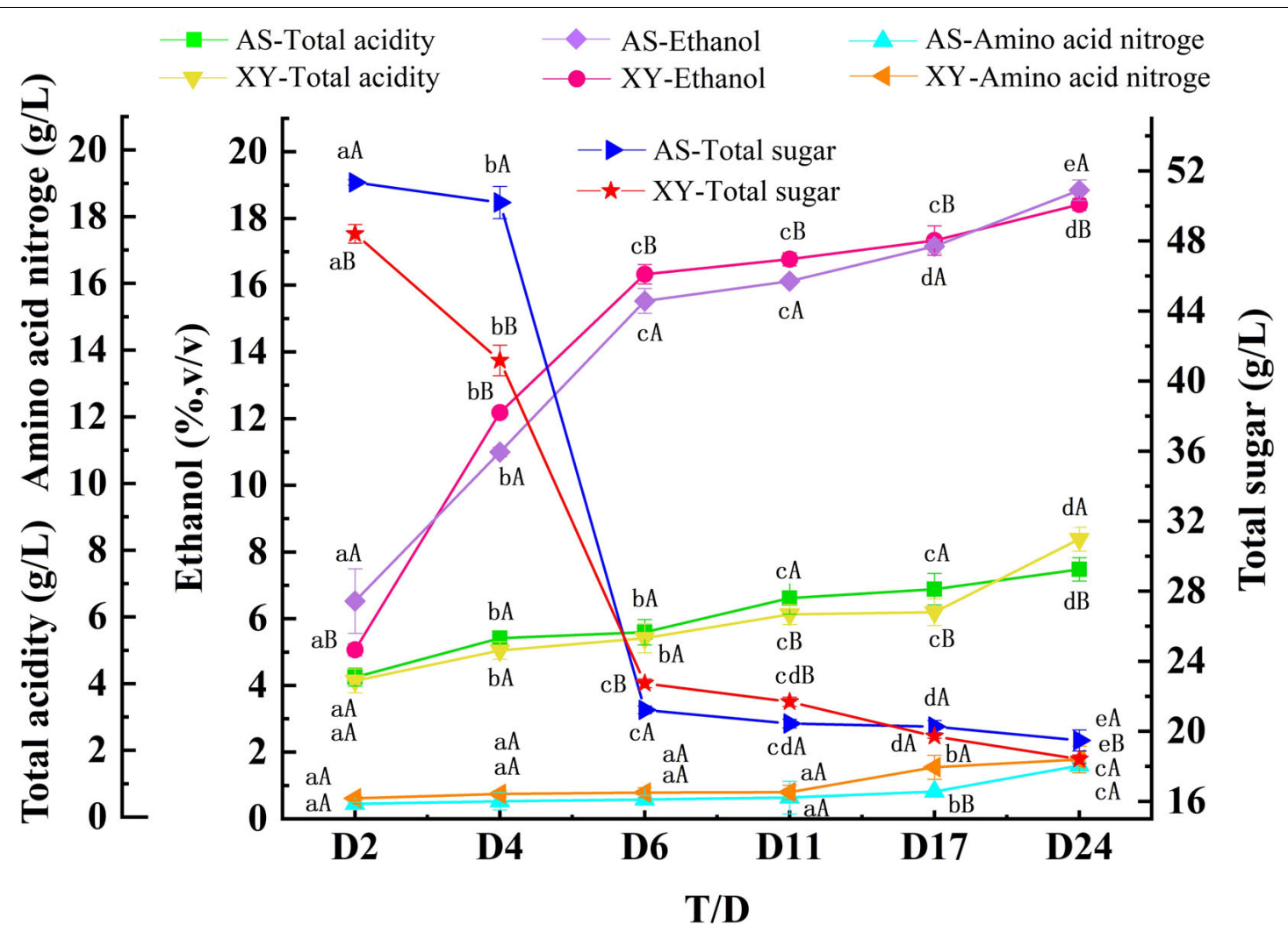

FIGURE 3 | Changes in physicochemical properties during fermentation of black glutinous rice wine using two different kinds of starters ( $n=3$ ). a-e: Different letters represent significant differences $(p<0.05)$ during fermentation. A,B: Different letters represent significant differences $(p<0.05)$ in two samples.

fluctuating trends in the two samples. After fermentation, AS had a higher concentration of aldehydes, while XY had a higher concentration of phenols. 2-Methoxy-phenol (P1) and benzaldehyde (Q2) were detected as characteristic compounds at the end of fermentation. In addition, a small number of other compounds (such as ketones, alkenes, alkanes) were detected in the BGRW (Figure 5F).

\section{PCA of the Major Metabolites During Different Brewing Stages}

The dynamics of the two wines during fermentation were visualized by PCA, using the concentrations of the compounds detected in the AS and XY samples as analytical variables. Figure 6A shows the relationships of the 53 flavor compounds in AS (including 7 organic acids and 46 volatile compounds), in which the first principal component (PC1) and second principal component (PC2) explained 44.64 and $14.40 \%$ of the total variance, respectively, reflecting $61.04 \%$ of the total flavor information in AS during brewing. Figure 6B shows the close associations among the 50 flavor compounds in XY (including 7 organic acids and 43 volatile compounds), in which PC1 and PC2 explained 56.10 and $21.00 \%$ of the total variance, respectively, reflecting $67.10 \%$ of the total flavor information in XY during brewing. The PCA scores can be used to quantify the difference in the distribution of the samples at different fermentation stages, which may be caused by the different starters. The flavors in AS changed significantly $(P<0.05)$ at the early stage of fermentation (2-6 days) and clustered after 6 days of fermentation, indicating that the flavors changed slowly during 6-24 days. By contrast, the dynamics of flavor components showed an opposite trend in $\mathrm{XY}$ in which the samples clustered during the first 2-6 days of fermentation and then changed significantly during 6-24 days.

\section{Correlations Between Microorganisms and Major Metabolites}

Finally, to determine the relationships among the metabolites and core microorganisms detected in the BGRW, correlation matrixes were constructed based on Pearson correlation coefficients. Here, we only focus on correlation coefficients showing significance at the 0.05 threshold level. The correlation coefficients showed very strong relationships in both samples (from 0.80 to 1 ). A total of 22 microbial genera were strongly correlated with 45 metabolites in AS (Figure 7A) and 17 microbial genera were strongly correlated with 44 metabolites in XY (Figure 7B). Key microorganisms were defined as those with at least 10 connections. A total of seven and four key microorganisms were detected in AS and XY, respectively, that affected the change of metabolites: Gluconobacter, Bacillus, Streptococcus, Lactobacillus, Lactococcus, Pediococcus, and Leuconostoc. Most of these microorganisms belong to the lactic acid bacteria (LAB), indicating that LAB play 

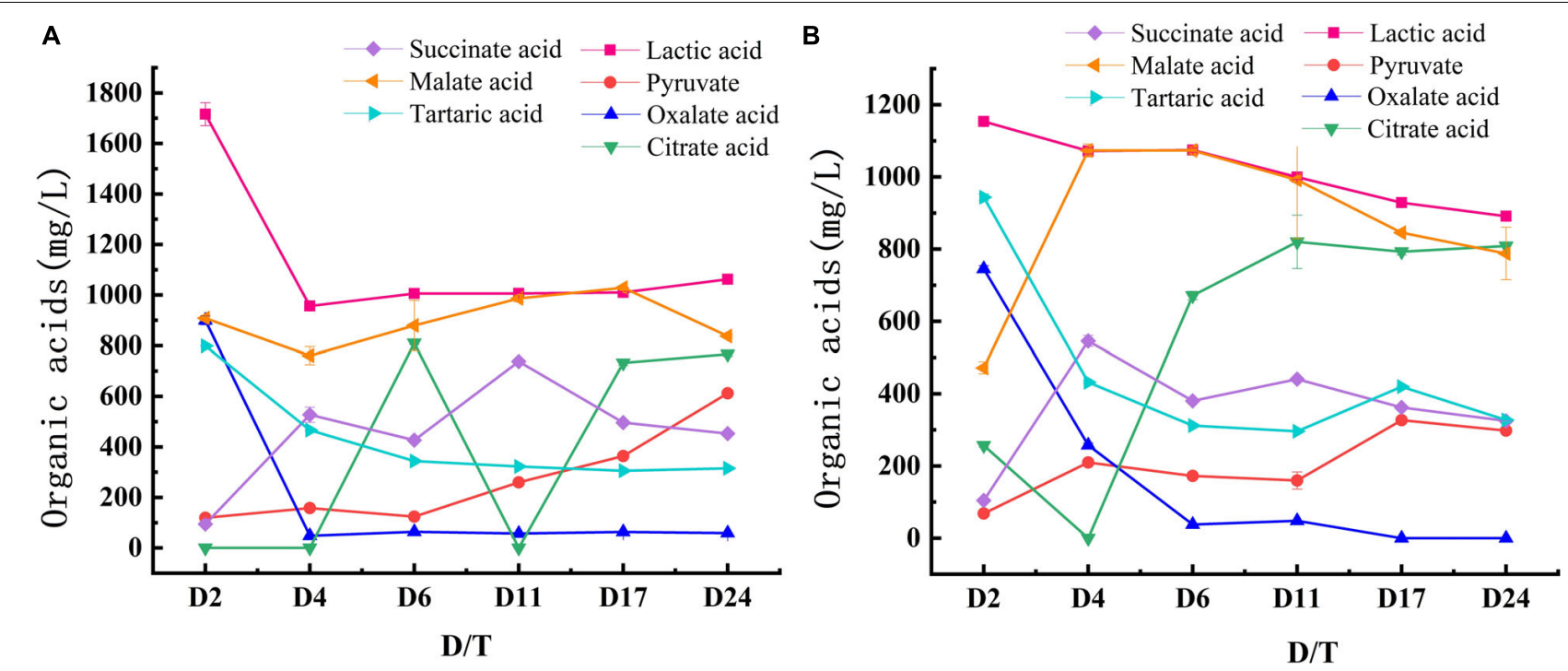

FIGURE 4 | Changes of organic acids content during fermentation of black glutinous rice wine with two kinds of starters. (A) AS and (B) XY.
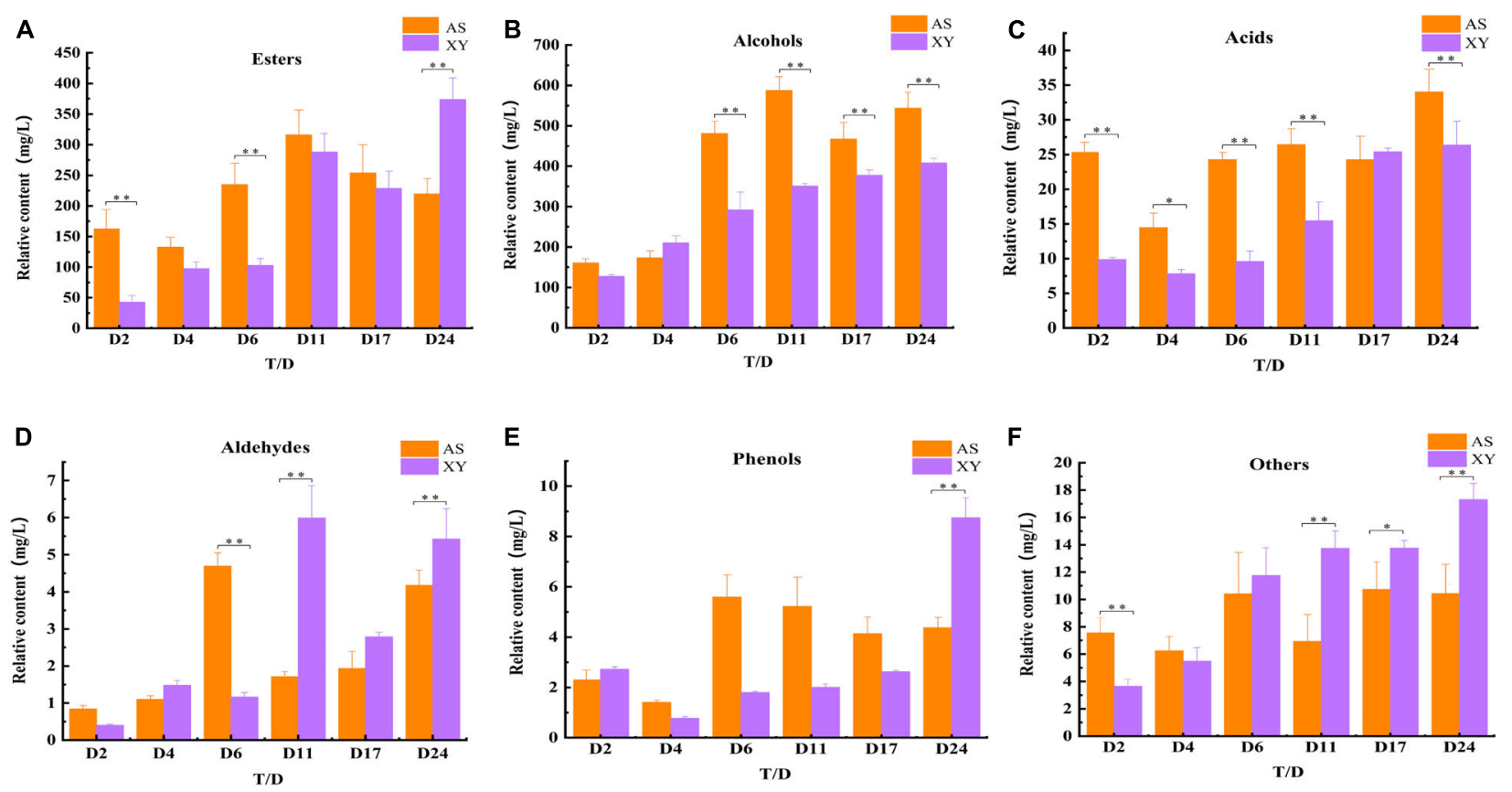

FIGURE 5 | Changes in alcohols (A), esters (B), acids (C), aldehydes (D), phenols (E), and others (F) during fermentation of black glutinous rice wine with AS or XY starter. $\left(n=3,{ }^{*} 0.01<p<0.05,{ }^{* *} p<0.01\right)$.

a vital role in BGRW fermentation. In addition to generating lactic acid, LAB may also promote the production of other flavors, such as alcohols, esters, and alkanes (Mukisa et al., 2016). Indeed, the abundance of LAB was positively correlated with numerous esters, alcohols, acids, and alkanes. However, Gluconobacter and Bacillus were positively correlated with butanoic acid (S3), lactic acid (S6), citric acid (C9), tartaric acid (S12), benzyl alcohol (C1), octanoic acid ethyl ester (Z4), ethyl 9-decenoate (Z4), 1-(1-ethoxyethoxy)-pentane (W1), and tridecane (W2).

\section{DISCUSSION}

This is the first study to comprehensively compare BGRW components produced by two different starters. The results provide new insight into changes in major metabolites and the microbial community throughout the brewing process. To explore the differences between the two starter samples, a comprehensive investigation of the microbial communities in different fermentation starters for GBRW 

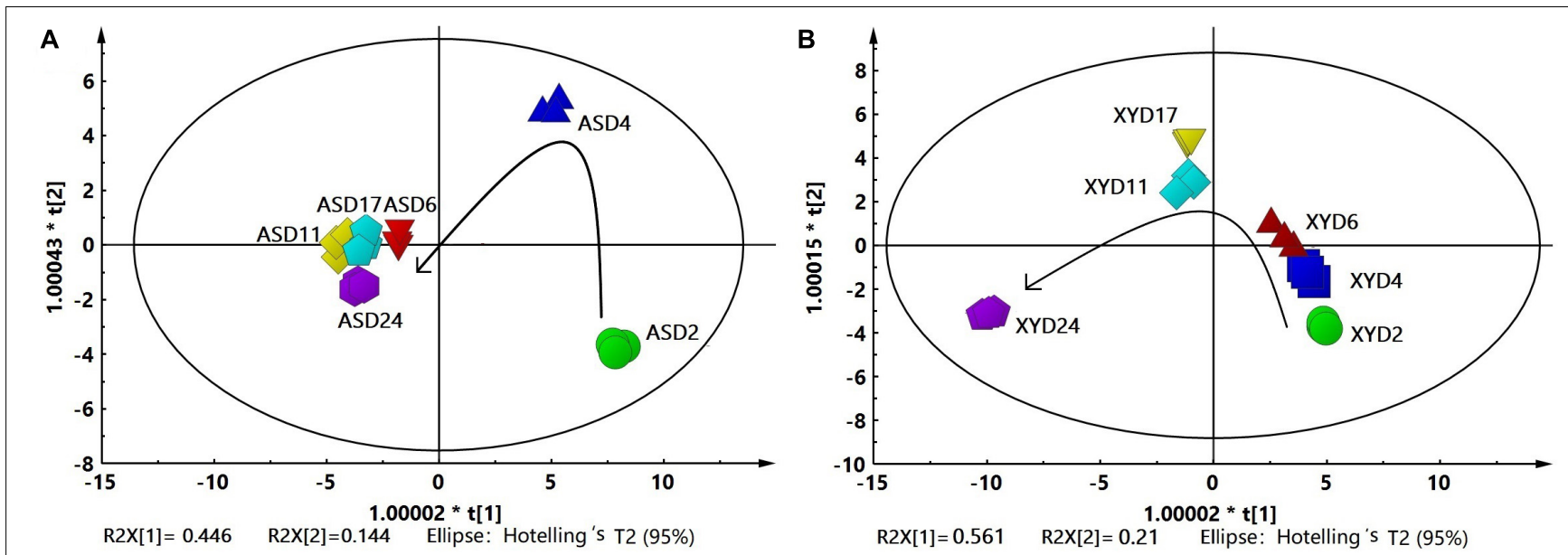

FIGURE 6 | Principal components analysis scores plots of major metabolites produced during the fermentation process of black glutinous rice wine with two kinds of starters. (A) AS and (B) XY.

was carried out using HTS technology. The results showed that the different starters clearly influenced the microbial diversity of the BGRW. A total of 18 bacteria and 4 fungi were identified as the dominant microorganisms in the two wine types. Pediococcus, Leuconostoc, Bacillus, Pantoea, and Lactobacillus were the main dominant bacterial genera in AS, whereas Leuconostoc, Pediococcus, Gluconobacter, Bacillus, and Acinetobacter were the main dominant bacterial genera in XY. In addition, several genera of the Lactobacillales order (including Lactococcus, Lactobacillus, Pediococcus, Streptococcus, Weissella, Enterococcus, and Leuconostoc) were detected throughout the 24-day fermentation. The same general result was reported in Hong Qu glutinous rice wine (Liu et al., 2018). Lactic acid bacteria are ubiquitous in fermented foods because they can withstand the low $\mathrm{pH}$ (Abriouel et al., 2006). As functional microorganisms, LAB play a key role in the fermentation of foods, and it has been shown to improve the sensory quality of rice wine by enhancing the digestibility of protein and increasing the availability of nutrients (Luana et al., 2014). Bacillus was reported to be the dominant bacterium in the fermentation of rice wine (Dijl and Jong, 1993; Lv et al., 2015; Liu et al., 2018), as it has the ability to secrete a large number of enzymes to extract conutrients for the production of other microorganisms, thus promoting the formation of the rice wine flavor (Liu et al., 2008). However, in the present study, the abundance of Bacillus decreased in conjunction with the increase in the abundance of Pediococcus and Lactococcus (Figure 1), indicating an antagonistic relationship between these genera. Koyanagi et al. (2013) reported that Bacillus might produce growth factors that are conducive to $\mathrm{LAB}$ growth during the postfermentation stage. Gluconobacter is a functional bacterium in fermented vinegar that can produce acetic acid and has also been found in Grenache grape wine (Portillo and Mas, 2016) and Chinese liquor (Wang et al., 2017) as the dominant microorganism. Gluconobacter is usually sensitive to alcohol and can be inhibited by high alcohol concentrations (Joyeux et al., 1984; Toit and Pretorius, 2002; González et al., 2005). This explains why its prevalence was greater in the early stage of fermentation, especially in the $\mathrm{XY}$ samples. Pantoea is common endophytes of rice (Megías et al., 2016) with antibacterial properties and is therefore often used as a biological control agent. In this study, the predominant bacterial genus Pantoea observed at day 0 might originate from the raw material of black glutinous rice and the starters. Similarly, Pantoea has been identified as the most dominant bacteria in Hong Qu starters (Coutinho and Venter, 2009; Walterson et al., 2014).

In addition to bacteria, rice wine fermentation also depends on mold and yeast. In particular, Rhizopus, Saccharomyces, and Saccharomycosis are popular genera in starters and are considered to be important functional microorganisms. Anshun had a higher proportion of Rhizopus, whereas XY had a higher proportion of yeast, which may be related to the different herbs added to the starter. Rhizopus, a strong amylase producer and frequently found in amylolytic fermentation starters for rice wine brewing (Dung et al., 2007; Thanh et al., 2008), was reported that the main fungi in $\mathrm{Yao} \mathrm{Qu}$ ( $\mathrm{Lv}$ et al., 2012). Saccharomyces is the most effective ethanol producer known so far (Vaughan-Martini and Martini, 1995), whereas Saccharomycopsis is a non-Saccharomyces cerevisiae with high amylase and glycosidase activities (Charoenchai et al., 1997). It has been reported that the yeast may act as a biocontrol agent against spoilage microorganisms increasing the shelf life of the product (Bora et al., 2016). Moreover, yeasts can stimulate LAB growth by supplying essential metabolites including pyruvate, amino acids, and vitamins (Jespersen, 2003). In both samples, the relative abundance of Rhizopus showed opposing dynamics to Saccharomyces and Saccharomycopsis (Figure 2), which may reflect the ability of Rhizopus to outcompete yeast for nutrients.

The physicochemical properties of a sample are typically used to determine the fermentation state and degree and can also reflect the microbial state in the fermented mash. After saccharification, the main reaction in mash involves the action of yeasts that use fermentable sugars to produce ethanol and other products. Thus, the total sugar and alcohol concentrations 

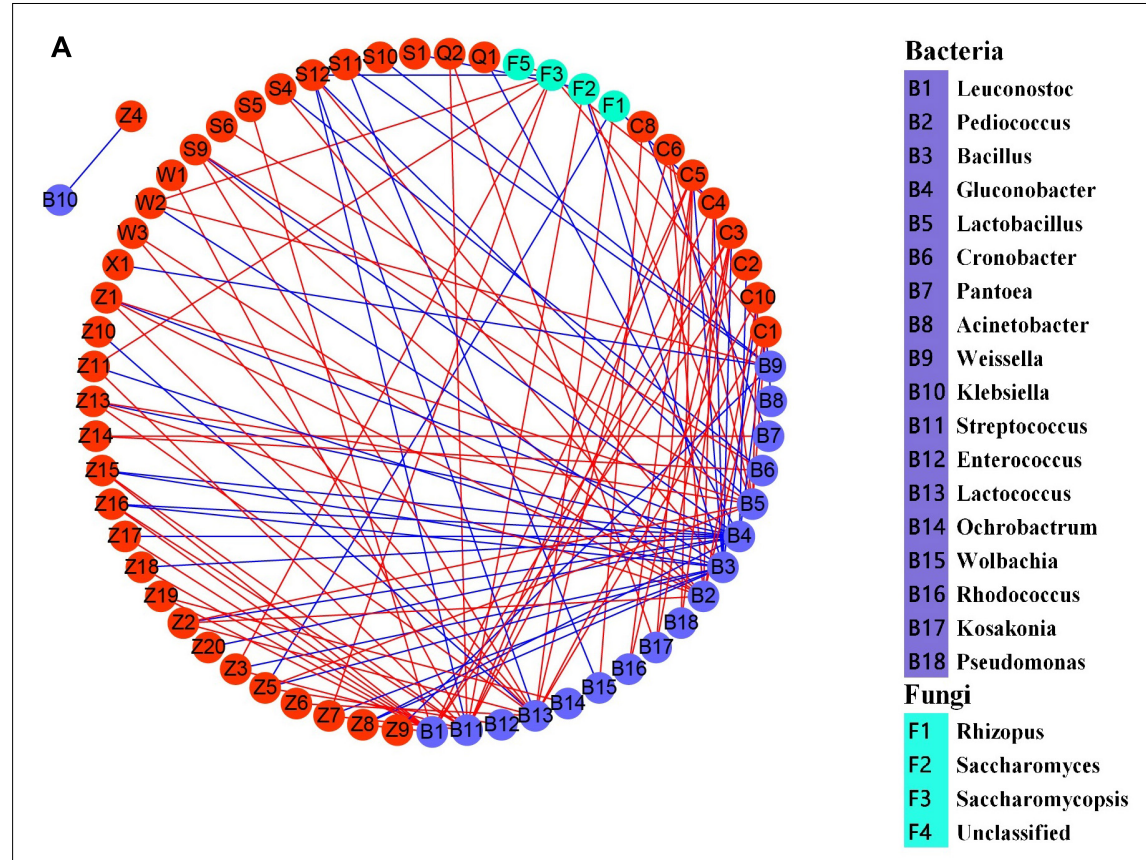

B

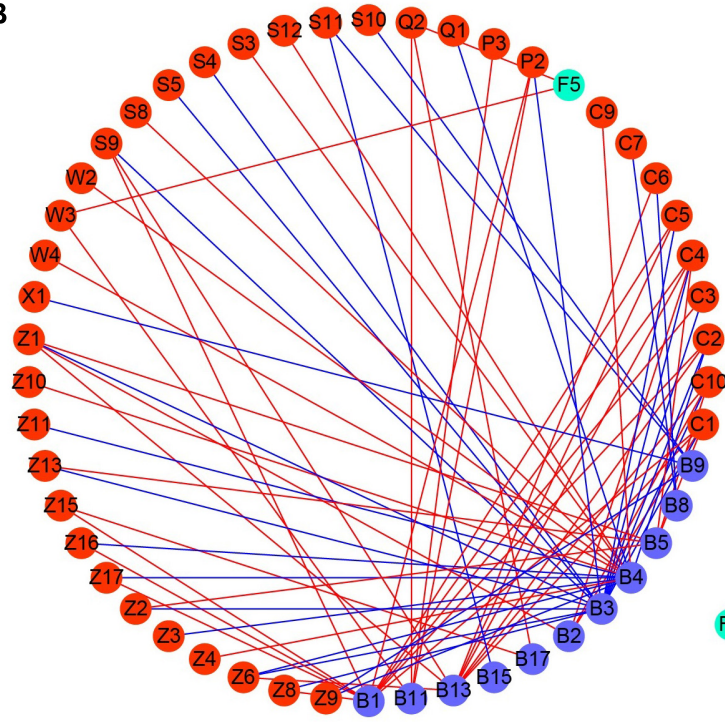

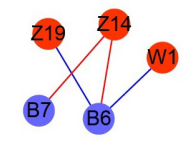

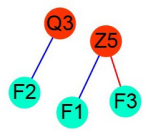

\begin{tabular}{l|l} 
Flavor Compound \\
C1 & Ethanol \\
C2 & 2-Methyl-1-Propanol \\
C3 & 1-Butanol \\
C4 & 3-Methyl-1-Butanol \\
C5 & 1-Hexanol \\
C6 & 2,3-Butanediol \\
C7 & 1-Octanol \\
C8 & 3-(Methylthio)-1-Propanol \\
C9 & Benzyl alcohol \\
C10 & Phenylethyl Alcohol \\
Q1 & Furfural \\
Q2 & Benzaldehyde \\
Q3 & 2,5-Dimethyl-Benzaldehyde \\
P1 & 2-Methoxy-Phenol \\
P2 & 4-Ethyl-2-methoxy-Phenol \\
P3 & 4-Ethyl-Phenol \\
Z1 & 3-Methyl-1-Butanol acetate \\
Z2 & Hexanoic acid ethyl ester \\
Z3 & Ethyl-lactate \\
Z4 & Octanoic acid ethyl ester \\
Z5 & Nonanoic acid ethyl ester \\
Z6 & 2-hydroxy-4-methyl-Pentanoic acid ethyl ester \\
Z7 & Isoamyl lactate \\
Z8 & Decanoic acid ethyl ester \\
Z9 & Octanoic acid 3-methylbutyl ester \\
Z10 & Ethyl 9-decenoate \\
Z11 & Butanedioic acid diethyl ester \\
Z12 & Benzeneacetic acid ethyl ester \\
Z13 & Acetic acid 2-phenylethyl ester \\
Z14 & Dodecanoic acid ethyl ester \\
Z15 & Pentadecanoic acid 3-methylbutyl ester \\
Z16 & Tetradecanoic acid ethyl ester \\
Z17 & Hexadecanoic acid ethyl ester \\
Z18 & Ethyl 9-hexadecenoate \\
Z19 & Ethyl Oleate \\
Z20 & Linoleic acid ethyl ester \\
W1 & 1-(1-Ethoxyethoxy)-Pentane \\
W2 & Tridecane \\
W3 & Tetradecane \\
W4 & Pentadecane \\
S1 & Acetic acid \\
S2 & 2-Methyl-Propanoic acid \\
S3 & Butanoic acid \\
S4 & Hexanoic acid \\
S5 & Octanoic acid \\
& \\
\end{tabular}

FIGURE 7 | Correlation network between major metabolites and microbial communities (based on Cytoscape software) during fermentation of black glutinous rice wine with two kinds of starters. (A) AS and (B) XY. Orange circles represent the flavor metabolites; purple circles represent bacteria, and cyan circles represent fungi. The red lines represent positive correlations, and the blue lines represent negative correlations.

in the AS and XY samples decreased and increased during fermentation, respectively (Figure 3). Establishment of a suitable environment in the early stage of fermentation causes the acid-producing microorganisms to multiply and produce acid metabolites, which leads to an increase of the total acid content. However, the growth of some acid-producing microorganisms is inhibited under higher acid and alcohol environments, resulting in a steady level of total acids during the later period of fermentation. The level of amino acid nitrogen directly affects the quality grade and overall flavor of wine. In line with a previous report showing that the content of amino acid nitrogen in rice wine is closely related to the amount of yeast and protein mass concentration in the mash (Pan et al., 2016), we observed similar trends in the dynamics of amino acid nitrogen and yeast during fermentation.

In addition to enhancing the flavor of rice wine, organic acids can also improve the intestinal tract and prevent fatigue. For example, malic acid can prevent fatigue, protect the liver, and enhance heart function (Liu et al., 2003), whereas citric acid can delay aging, eliminate fatigue, and reduce blood pressure (Feng et al., 2010). Correlation analysis showed that five and three microorganisms in AS and XY, respectively, were significantly 
correlated with the formation of organic acids. Lactic acid, as the primary organic acid in the two samples, can be esterified to form ethyl lactate, which is the main aromatic body of rice wine (Gao et al., 2014). Indeed, the high level of lactic acid in BGRW is associated with the abundance of LAB. Thus, a higher content of lactic acid was found in XY compared with AS, which likely reflects the larger proportion of LAB in XY. In addition, a positive correlation between lactic acid and Cronobacter was observed. Notably, Cronobacter is a foodborne pathogen, most commonly detected in infant formula. Fortunately, the BGRW brewing process includes a sterilization step $\left(80^{\circ} \mathrm{C}\right.$ for $\left.30 \mathrm{~min}\right)$, which can kill most pathogenic bacteria, thereby improving the safety of rice wine. Malic acid, the second dominant organic acid, has been reported to be related to yeast and Aspergillus (Peleg et al., 1988; Pines et al., 1996), but no relationship was found between them. However, our study suggested that Weissella and Wolbachia were correlated significantly with malic acid, in which Weissella was identified as one of the dominant LAB in Dombea, a Cambodian traditional stater (Sokny et al., 2018). But its abundance begins to decrease after 2 days in two samples. In AS, Lactobacillus, Pediococcus, and Lactococcus were all positively correlated with citric acid, and Bacillus contributed to an increase in tartaric acid. Among these genera, Lactococcus produces acids by using carbohydrates, Pediococcus produces acids by fermenting sugars (Ren et al., 2019), and Bacillus has strong enzymatic activity and can produce abundant metabolites to synthesize various organic acids. Gluconobacter was reported that can promote the formation of acids, alcohols, and esters in rice wine fermentation (Saichana et al., 2014). In this study, Gluconobacter was positively correlated with butanoic, oxalic, and tartaric acids in XY. Because Gluconobacter is inhibited by higher alcohol levels (Joyeux et al., 1984; Toit and Pretorius, 2002; González et al., 2005), an increase in alcohol content during fermentation also affected the butanoic, oxalic, and tartaric acids content.

There were minimal differences in the volatile metabolites between the two starters. Correlation analysis showed that about twice the numbers of microorganisms were involved in the formation of alcohols in AS compared to those in XY. Ethanol was the most abundant alcohol detected in the two wines, which can be formed from acetaldehyde produced by LAB (Engels et al., 1997). Lactobacillus, Pediococcus, Leucoconstoc, Lactococcus, and Saccharomycopsis are involved in ethanol formation, all of which are LAB except for Saccharomycopsis. Limtong et al. (2002) reported that Saccharomycopsis contributes to alcohol production. Furthermore, the cooperative fermentation of Saccharomycopsis and Saccharomyces can adjust the alcohol content to a certain extent and improve the aroma quality of wine (Liu et al., 2016). The alcohols 2-methyl-1-propanol, 3methyl-1-butanol, and phenylethyl are the metabolites of valine, leucine, and phenylalanine, respectively, and are the typical flavor compounds in fermented alcoholic beverages that also constitute the overall sensory complexity of rice wine (Chen et al., 2010; Hye-Jung et al., 2013). In AS, the level changes of 2-methyl-1-propanol, 3-methyl-1-butanol, and phenylethanol were related to the dynamics in the abundance of Leuconostoc, Lactobacillus, Pedicoccus, and Streptococcus. In XY, although the production of 2-methyl-1-propanol and phenylethanol was strongly impacted by Leuconostoc, none of the microorganisms detected was associated with 3-methyl-1-butanol. 2,3-Butanediol is one of the few aromatic polyols in wines, with a creamy and buttery aroma. Early studies showed that Bacillus in starter could produce 2,3-butanediol ( $\mathrm{Wu}$ and $\mathrm{Xu}, 2012$ ). We also demonstrated that $\mathrm{LAB}$ contributed to the formation of 2,3-butanediol, which was also detected as the characteristic compound of XY after fermentation.

Esters have been reported to endow rice wine with fruity and floral odors (Fang et al., 2015). Most of the esters detected in the present study were ethyl esters, which is in line with previous studies (Chen and Xu, 2010; Yang et al., 2017b). Ethyl ester is usually produced by the esterification of fatty acids and ethanol during fermentation and aging (Cheong et al., 2010; Yu et al., 2014), mainly due to yeast and other microorganisms metabolism (Fan and Qian, 2005). Correlation analysis indicated that Pantoea, Leuconostoc, Lactobacillus, Streptococcus, Pediococcus, Enterococcus, Saccharomycopsis, Lactococcus, Ochrobactrum, and Gluconobacter were positively correlated with esters (Figure 7). Octanoic acid, decanoic acid, and hexadecanoic acid ethyl esters were the main esters identified in this study. Decanoic acid ethyl ester has a brandy, fruity, and grape-like aroma; octanoic acid ethyl ester has a floral and pear aroma; and hexadecanoic acid ethyl ester has an apple aroma. 4-Ethylphenol and 4ethyl 2-methoxyphenol were the main phenolic compounds detected in AS, which are the main aroma substances of red wine that are formed by the metabolism of $\rho$-coumaric acid and ferulic acid, respectively (Pollnitz et al., 2000). Chen and $\mathrm{Xu}$ (2010) also reported that 4-ethyl 2-methoxyphenol was the most abundant phenolic compound in Chinese rice wine fermented with wheat $\mathrm{Qu}$ with the highest odor activity value, exhibiting characteristics of a phenolic, clove, and smoke-like flavor. Aspergillus, Streptococcus, Lactobacillus, and Leuconostoc were positively correlated with 4-ethylphenol and 4-ethyl 2-methoxyphenol (Figure 7). 2-Methoxy-phenol was the characteristic phenol detected in XY, and its content was significantly higher than that of AS. This may be due to higher abundance of related microorganisms in XY than in AS. Benzaldehyde has a typical almond and cherry odor (Jung et al., 2014), and Aspergillus and Streptococcus play the greatest roles in its formation. Yoshizaki et al. (2010) stated that Aspergillus is related with the metabolites of aldehydes. Thus, the content of benzaldehyde was higher at the end of fermentation in XY, which may be related to the higher abundance of Aspergillus. Acetoin is an important physiological metabolite that is excreted by many microorganisms and serves as a quality indicator of fermented products (Xiao and Xu, 2007). Members of the genera Acetobacter, Lactococcus, Klebsiella, Enterobacter, and Bacillus are considered as synthetic acetoin bacteria during fermentation (Molinari et al., 2003; Chen et al., 2010). Although we did not detect a relationship between the microorganisms and acetoin, it is worth noting that correlation is not equal to causation. Overall, our results reflect the highly complex relationship between microorganisms and flavor substances during BGRW fermentation and indicate that the production of flavor substances is likely the result of the synergistic effect of microorganisms and biochemical reactions. 


\section{CONCLUSION}

Tracking and comparison of the physicochemical properties and microbial community structures, along with their corresponding flavors, during BGRW fermentation with two different starters showed that the physical and chemical indicators and flavors were strongly affected by the microbial community. Pediococcus, Leuconostoc, Bacillus, Gluconobacter, and Lactobacillus were the dominant bacteria, whereas Rhizopus, Saccharomyces, and Saccharomycopsis were the predominant fungi detected during fermentation. The potential role of microorganisms in flavor formation was also demonstrated through the Pearson correlation coefficient. Lactic acid bacteria were identified as the key microorganisms contributing to the production of flavor. These results have a certain reference value for the quality improvement and technical optimization of BGRW production. Nevertheless, further studies should be devoted to validating the association between the core microorganisms identified and the specific flavor components using multiomics approaches, including metagenomics, metaproteomics, and metatranscriptomics.

\section{DATA AVAILABILITY STATEMENT}

All sequencing data have been deposited at the Sequence Read Archive of the National Center for Biotechnology Information (NCBI), with SRA accession number: the accession number of bacteria and fungi are PRJNA597320 and PRJNA597372, respectively.

\section{REFERENCES}

Abriouel, H., Omar, N. B., López, R. L., Amero, M. M., Keleke, S., and Gálvez, A. (2006). Culture-independent analysis of the microbial composition of the African traditional fermented foods poto poto and dégué by using three different DNA extraction methods. Int. J. Food Microbiol. 111, 228-233. doi: 10.1016/j.ijfoodmicro.2006.06.006

Atsushi, T., Miyuki, K., Koji, T., Toshiki, E., and Takashi, K. (2018). Robust domination of lactobacillus sakei in microbiota during traditional japanese sake starter Yamahai-Moto fermentation and the accompanying changes in metabolites. Curr. Microbiol. 75, 1498-1505. doi: 10.1007/s00284-018-1551-8

Bora, S. S., Keot, J., Das, S., Sarma, K., and Barooah, M. (2016). Metagenomics analysis of microbial communities associated with a traditional rice wine starter culture (Xaj-pitha) of Assam. India. Biotech 6, 1-13. doi: 10.1007/s13205-0160471-1

Buée, M., Reich, M., Murat, C., and Morin, E. (2009). 454 Pyrosequencing analyses of forest soils reveal an unexpectedly high fungal diversity. New Phytol. 184, 449-456. doi: 10.1111/j.1469-8137.2009.03003.x

Cai, H., Zhang, T., Zhang, Q., Luo, J., Cai, C., and Mao, J. (2018). Microbial diversity and chemical analysis of the starters used in traditional Chinese sweet rice wine. Food Microbiol. 73, 319-326. doi: 10.1016/j.fm.2018.02.002

Caporaso, J. G., Kuczynski, J., Stombaugh, J., Bittinger, K., Bushman, F. D., and Costello, E. (2010). QIIME allows analysis of high-throughput community sequencing data. Nat. Methods 7, 335-336. doi: 10.1038/nmeth.f.303

Charoenchai, C., Fleet, G. H., Henschke, P. A., and Todd, B. E. N. T. (1997). Screening of non-Saccharomyces wine yeasts for the presence of extracellular hydrolytic enzymes. Aust. J. Grape Wine Res. 3, 2-8. doi: 10.1111/j.1755-0238. 1997.tb00109.x

Chen, J. C., Chen, Q., Guo, Q., Ruan, S., Ruan, H., and He, G. (2010). Simultaneous determination of acetoin and tetramethylpyrazine in traditional vinegars by

\section{AUTHOR CONTRIBUTIONS}

LJ and WS contributed the experimental design. LJ performed the statistical analysis and wrote the manuscript. LJ, WS, YiM, and YuM contributed to manuscript revision, read and approved the submitted version.

\section{FUNDING}

The research was financially supported by the National Natural Science Foundation of China under Grant No. 31860441; and Guangzhou Science and Technology Plan Project under Grant No. 201604020001.

\section{ACKNOWLEDGMENTS}

The authors thank Biomarker Technologies Co, Ltd. for technical assistance in carrying out the analyses. The authors also thank Editage (www.editage.cn) for English language editing.

\section{SUPPLEMENTARY MATERIAL}

The Supplementary Material for this article can be found online at: https://www.frontiersin.org/articles/10.3389/fmicb. 2020.00593/full\#supplementary-material

HPLC method. Food Chem. 122, 1247-1252. doi: 10.1016/j.foodchem.2010.0 3.072

Chen, S., and $\mathrm{Xu}, \mathrm{Y}$. (2010). The influence of yeast strains on the volatile flavour compounds of chinese rice wine. J. Inst. Brew. 116, 190-196. doi: 10.1002/j. 2050-0416.2010.tb00417.x

Cheong, K. W., Tan, C. P., Mirhosseini, H., Hamid, N. S. A., Osman, A., and Basri, M. (2010). Equilibrium headspace analysis of volatile flavor compounds extracted from soursop (Annona muricata) using solid-phase microextraction. Food Res. Int. 43:1276. doi: 10.1016/j.foodres.2010.03.001

Coutinho, T. A., and Venter, S. N. (2009). Pantoea ananatis: an unconventional plant pathogen. Mol. Plant Pathol. 10, 325-335. doi: 10.1111/j.1364-3703.2009. 00542.x

Delgado, S., Peixoto, R. S., Leite, A. M. O., Alegria, A., Rachid, C. T. C. C., and Mayo, B. (2014). Impact of next generation sequencing techniques in food microbiology. Curr. Genomics 15, 293-309. doi: 10.2174/ 1389202915666140616233211

Dijl, J. M. V., and Jong, A. D. (1993). Protein secretion in Bacillus subtilis. DNA Transfer Gene Expression Microorg 3, 251-262.

Dung, N. T. P., Rombouts, F. M., and Nout, M. J. R. (2007). Characteristics of some traditional Vietnamese starch-based rice wine fermentation starters (men). LWT-Food Sci. Technol. 40:135. doi: 10.1016/j.lwt.2005.08.004

Edgar, R. C., Haas, B. J., Clemente, J. C., Quince, C., and Knight, R. (2011). UCHIME improves sensitivity and speed of chimera detection. Bioinformatics 27:2194. doi: 10.1093/bioinformatics/btr381

Engels, W. J. M., Dekker, R., Jong, C. D., Neeter, R., and Visser, S. A. (1997). A comparative study of volatile compounds in the water-soluble fraction of various types of ripened cheese. Int. Dairy J. 7, 255-263. doi: 10.1016/S09586946(97)00003-4

Fan, W., and Qian, M. C. (2005). Headspace solid phase microextraction and gas chromatography-olfactometry dilution analysis of young and aged Chinese 
"Yanghe Daqu" liquors. J. Agric. Food Chem. 53, 7931-7938. doi: 10.1021/ jf051011k

Fang, R. S., Dong, Y., Chen, F., and Chen, Q. (2015). Bacterial diversity analysis during the fermentation processing of traditional chinese yellow rice wine revealed by $16 \mathrm{~S}$ rDNA 454 pyrosequencing. J. Food Sci. 80, M2265-M2271. doi: 10.1111/1750-3841.13018

Feng, A., Zhao, W., Bai, W., and Pang, J. (2010). Determination of organic acids in different rice wine. China Brew. 8, 144-146.

Fimognari, C., Lenzi, M., and Hrelia, P. (2008). Chemoprevention of cancer by isothiocyanates and anthocyanins: mechanisms of action and structure-activity relationship. Curr. Med. Chem. 15, 440-447. doi: 10.2174/092986708783503168

Gao, W., Fan, W., and Xu, Y. (2014). Characterization of the key odorants in light aroma type chinese liquor by gas chromatography-olfactometry, quantitative measurements, aroma recombination, and omission studies. J. Agric. Food Chem. 62, 5796-5804. doi: 10.1021/jf501214c

GB/T 13662, (2018). Chinese Rice Wine. Beijing: Code of China.

González, Á, Hierro, N., Poblet, M., Mas, A., and Guillamón, J. M. (2005). Application of molecular methods to demonstrate species and strain evolution of acetic acid bacteria population during wine production. Int. J. Food Microbiol. 102, 295-304. doi: 10.1016/j.ijfoodmicro.2004.11.020

Hong, X., Chen, J., Liu, L., Wu, H., Tan, H., and Xie, G. (2016). Metagenomic sequencing reveals the relationship between microbiota composition and quality of Chinese Rice Wine. Sci. Rep. 6:26621. doi: 10.1038/srep26621

Hou, D. (2003). Potential mechanisms of cancer chemoprevention by anthocyanins. Curr. Mol. Med. 3, 149-159. doi: 10.2174/1566524033361555

Hye-Jung, P., Sang, L., Sang, S., and Young-Suk, K. (2013). Characterization of volatile components in makgeolli, a traditional korean rice wine, with or without pasteurization, during storage. Molecules 18, 5317-5325. doi: 10.3390/ molecules 18055317

Jespersen, L. (2003). Occurrence and taxonomic characteristics of strains of Saccharomyces cerevisiae predominant in African indigenous fermented foods and beverages. FEMS Yeast Res. 3, 191-200. doi: 10.1016/s1567-1356(02)001 85-x

Joyeux, A., Lafon-Lafourcade, S., and Ribéreau-Gayon, P. (1984). Evolution of acetic acid bacteria during fermentation and storage of wine. Appl. Environ. Microbiol. 48, 153-156. doi: 10.1007/BF02386244

Jung, H., Lee, S. J., Lim, J. H., Kim, B. K., and Park, K. J. (2014). Chemical and sensory profiles of makgeolli, Korean commercial rice wine, from descriptive, chemical, and volatile compound analyses. Food Chem. 152, 624-632. doi: 10.1016/j.foodchem.2013.11.127

Junka, N., Rattanamechaiskul, C., and Wongs-Aree, C. (2016). Free fatty acid deformation of treated black glutinous rice during storage by fluidization drying. J. Food Process Eng. 40, e12427. doi: 10.1111/jfpe.12427

Koyanagi, T., Nakagawa, A., Kiyohara, M., Matsui, H., Yamamoto, K., and Barla, F. (2013). Pyrosequencing analysis of microbiota in Kaburazushi, a traditional medieval sushi in Japan. Biosci. Biotechnol. Biochem. 77, 2125-2130. doi: 10. 1271/bbb. 130550

Lee, S., Lee, S., Singh, D., Oh, J. Y., and Lee, C. H. (2016). Comparative evaluation of microbial diversity and metabolite profiles in doenjang, a fermented soybean paste, during the two different industrial manufacturing processes. Food Chem. 221, 1578-1586. doi: 10.1016/j.foodchem.2016.10.135

Limtong, S., Sintara, S., Suwannarit, P., and Lotong, N. (2002). Yeast diversity in Thai traditional alcoholic starter. Kasetsart. J. Nat. Sci. 36, 149-158.

Liu, G. H., Liu, B., Lin, N. Q., and Lin, Y. Z. (2008). Phyletic evolution and taxonomic characteristics of Bacillus. Fujian J. Agric. Sci. 4, 436-499. doi: 10. 19303/j.issn.1008-0384.2008.04.021

Liu, J., Jiang, L., Zhao, X., Li, P., Tian, Y., and Zhang, J. (2003). Research and Application on L-Malic Acid. China Food Addit. 3, 53-56. doi: 10.3969/j.issn. 1006-2513.2003.03.016

Liu, M., Tang, Y., Zhao, K., Liu, Y., Guo, X., Ren, D., et al. (2017). Determination of the fungal community of pit mud in fermentation cellars for Chinese strongflavor liquor, using DGGE and Illumina MiSeq sequencing. Food Res. Int. 91, 80-87. doi: 10.1016/j.foodres.2016.11.037

Liu, P. T., Lu, L., Duan, C., and Yan, G. (2016). The contribution of indigenous nonSaccharomyces wine yeast to improved aromatic quality of Cabernet Sauvignon wines by spontaneous fermentation. LWT-Food Sci. Technol. 71, 356-363. doi: 10.1016/j.lwt.2016.04.031

Liu, Z., Wang, Z., Lv, X., Zhu, X., Chen, L., and Ni, L. (2018). Comparison study of the volatile profiles and microbial communities of Wuyi Qu and Gutian $\mathrm{Qu}$, two major types of traditional fermentation starters of Hong Qu glutinous rice wine. Food Microbiol. 69:105. doi: 10.1016/j.fm.2017.07.019

Luana, N., Rossana, C., Curiel, J. A., Kaisa, P., Marco, G., and Rizzello, C. (2014). Manufacture and characterization of a yogurt-like beverage made with oat flakes fermented by selected lactic acid bacteria. Int. J. Food Microbiol. 185, 17-26. doi: 10.1016/j.ijfoodmicro.2014.05.004

Lv, X., Weng, X., Zhang, W., Rao, P., and Ni, L. (2012). Microbial diversity of traditional fermentation starters for Hong Qu glutinous rice wine as determined by PCR-mediated DGGE. Food Control 28, 426-434. doi: 10.1016/j.foodcont. 2012.05.025

Lv, X. C., Chen, Z. C., Rui, B. J., Zhi, B. L., Wen, Z., and Shao, J. C. (2015). Microbial community structure and dynamics during the traditional brewing of Fuzhou Hong Qu glutinous rice wine as determined by culture-dependent and cultureindependent techniques. Food Control 57, 216-224. doi: 10.1016/j.foodcont. 2015.03.054

Lv, X. C., Jiang, Y., Liu, J., Guo, W., Liu, Z., and Zhang, W. (2017). Evaluation of different PCR primers for denaturing gradient gel electrophoresis (DGGE) analysis of fungal community structure in traditional fermentation starters used for Hong Qu glutinous rice wine. Int. J. Food Microbiol. 255, 58-65. doi: 10.1016/j.ijfoodmicro.2017.05.010

Magoc, T., and Salzberg, S. L. (2011). FLASH: fast length adjustment of short reads to improve genome assemblies. Bioinformatics 27, 2957-2963. doi: 10.1093/ bioinformatics/btr507

Megías, E., Megías, M., Ollero, F. J., and Hungria, M. (2016). Draft genome sequence of pantoea ananatis strain AMG521, a rice plant Growth-Promoting bacterial endophyte isolated from the guadalquivir marshes in southern spain. Genome Announc. 4, e1615-e1681. doi: 10.1128/genomeA.01681-15

Miya, M., Sato, Y., Fukunaga, T., Sado, T., Poulsen, J. Y., Minamoto, T., et al. (2015). MiFish, a set of universal PCR primers for metabarcoding environmental DNA from fishes: detection of more than 230 subtropical marine species. R. Soc. Open Sci. 2:150088. doi: 10.1098/rsos.150088

Molinari, F., De Faveri, D., Torre, P., Perego, P., and Converti, A. (2003). Carbon material balances and bioenergetics of 2,3-butanediol bio-oxidation by Acetobacter hansenii. Enzyme Microb. Technol. 33, 708-719. doi: 10.1016/ S0141-0229(03)00218-7

Mukisa, I. M., Byaruhanga, Y. B., Muyanja, C. M. B. K., Langsrud, T., and Narvhus, J. A. (2016). Production of organic flavor compounds by dominant lactic acid bacteria and yeasts from Obushera, a traditional sorghum malt fermented beverage. Food Sci. Nutr. 5, 702-712. doi: 10.1002/fsn3.450

Nie, Z., Zheng, Y., Du, H., Xie, S., and Wang, M. (2015). Dynamics and diversity of microbial community succession in traditional fermentation of Shanxi aged vinegar. Food Microbiol. 47, 62-68. doi: 10.1016/j.fm.2014.11.006

Pan, H., Cao, H., Shi, H., Lu, J., and Xie, G. (2016). Effect of post-fermentation technology on amino acid nitrogen generating during chinese rice wine brewing. J. Food Sci. Biotechnol. 35, 144-150. doi: 10.3969/j.issn.1673-1689. 2016.02.005

Peleg, Y., Stieglitz, B., and Goldberg, I. (1988). Malic acid accumulation by Aspergillus flavus. Appl. Microbiol. Biotechnol. 28, 69-75. doi: 10.1007/ BF00250501

Pines, O., Even-Ram, S., Elnathan, N., Battat, E., Aharonov, O., and Gibson, D. (1996). The cytosolic pathway of L-malic acid synthesis in Saccharomyces cerevisiae: the role of fumarase. Appl. Microbiol. Biotechnol. 46, 393-399. doi: 10.1007/BF00166235

Pollnitz, A. P., Pardon, K. H., and Sefton, M. A. (2000). Quantitative analysis of 4-ethylphenol and 4 ethylguaicol in red wine. J. Chromatogr. 874, 101-109. doi: 10.1016/S0021-9673(00)00086-8

Portillo, M. D. C., and Mas, A. (2016). Analysis of microbial diversity and dynamics during wine fermentation of Grenache grape variety by high-throughput barcoding sequencing. LWT Food Sci. Technol. 72, 317-321. doi: 10.1016/j.lwt. 2016.05.009

Qi, Q., Li, J., Mu, Y., Qiu, S., and Su, W. (2018). Experimental study on the effect of black glutinous rice health wine on tonifying kidney in mice. J. Biobased Mater. Bioenergy. 12, 148-152. doi: 10.1166/jbmb.2018.1752

Ren, Q., Sun, L., Wu, H., Wang, Y., Wang, Z., and Zheng, F. (2019). The changes of microbial community and flavor compound in the fermentation process of Chinese rice wine using Fagopyrum tataricum grain as feedstock. Sci. Rep. 9:3365. doi: 10.1038/s41598-019-40337-8

Saichana, N., Matsushita, K., Adachi, O., Frébort, I., and Frébortová, J. (2014). Acetic acid bacteria: a group of bacteria with versatile biotechnological 
applications. Biotechnol. Adv. 33, 1260-1271. doi: 10.1016/j.biotechadv.2014.1 2.001

Shen, Y., Jin, L., Xiao, P., Lu, Y., and Bao, J. (2009). Total phenolics, flavonoids, antioxidant capacity in rice grain and their relations to grain color, size and weight. J. Cereal Sci. 49, 106-111. doi: 10.1016/j.jcs.2008.07.010

Sokny, L., Hasika, M., Cédric, T., Bernard, T., Georges, D., and Marie, L. (2018). Impact of microbial composition of Cambodian traditional dried starters (Dombea) on flavor compounds of rice wine: combining amplicon sequencing with HP-SPME-GC-MS. Front. Microbiol. 9:894. doi: 10.3389/fmicb.2018. 00894

Su, W., Mu, Y., Qi, Q., Mu, Y., and Yu, X. (2018). Quality analysis of black glutinous rice wine and black glutinous rice health wine. China Brew. 37, 186-191. doi: 10.11882/j.issn.0254-5071.2018.12.035

Su, W., Qi, Q., Zhao, X., Mu, Y., and Qiu, S. (2017). Analysis of flavoring substances in black glutinous rice wine by electronic tongue combined with GC/MS. Liquor Making Sci. Technol. 9, 102-106. doi: 10.13746/j.njkj.201711

Su, W., Qi, Q., Zhao, X., Mu, Y. C., Qiu, S. Y., and Bai, W. D. (2015). Variation and biological activity of polyphenols during brewing process of black glutinous rice wine. Guizhou Agric. Sci. 45, 112-117.

Tang, Q., He, G., Huang, J., Wu, C., Jin, Y., and Zhou, R. (2019). Characterizing relationship of microbial diversity and metabolite in sichuan xiaoqu. Front. Microbiol. 10:696. doi: 10.3389/fmicb.2019.00696

Thanh, V. N., Le, T. M., and Tuan, D. A. (2008). Microbial diversity of traditional Vietnamese alcohol fermentation starters (banh men) as determined by PCR-mediated DGGE. Int. J. Food Microbiol. 128, 268-273. doi: 10.1016/j. ijfoodmicro.2008.08.020

Toit, W. J. D., and Pretorius, I. S. (2002). The Occurrence, control and esoteric effect of acetic acid bacteria in winemaking. Ann. Microbiol. 52, 155-179. doi: 10.1081/ABIO-120016191

Vaughan-Martini, A., and Martini, A. (1995). Facts, myths and legends on the prime industrial microorganism. J. Ind. Microbiol. 14, 514-522. doi: 10.1007/ bf01573967

Walterson, A. M., Smith, N. D. D., John, S., and Mark, G. (2014). Identification of a pantoea biosynthetic cluster that directs the synthesis of an antimicrobial natural product. PLoS One 9:e96208. doi: 10.1371/journal.pone.009 6208

Wang, X., Du, H., and Xu, Y. (2017). Source tracking of prokaryotic communities in fermented grain of Chinese strong-flavor liquor. Int. J. Food Microbiol. 244, 27-35. doi: 10.1016/j.ijfoodmicro.2016.12.018
Wu, Q., and Xu, Y. (2012). Transcriptome profiling of Heat-Resistant strain bacillus licheniformis CGMCC3962 producing maotai flavor. J. Agric. Food Chem. 60, 2033-2038. doi: 10.1021/jf204270h

Xiao, Z., and Xu, P. (2007). Acetoin metabolism in bacteria. Crit. Rev. Microbiol. 33, 127-140. doi: 10.1080/10408410701364604

Yang, Y., Xia, Y., Wang, G., Yu, J., and Ai, L. (2017a). Effect of mixed yeast starter on volatile flavor compounds in Chinese rice wine during different brewing stages. LWT Food Sci. Technol. 78, 373-381. doi: 10.1016/j.lwt.2017.01.007

Yang, Y., Xia, Y., Wang, G., Zhang, H., Xiong, Z., and Yu, J. (2017b). Comparison of oenological property, volatile profile, and sensory characteristic of Chinese rice wine fermented by different starters during brewing. Int. J. Food Prop. 20, 1-17. doi: 10.1080/10942912.2017.1325900

Yoshizaki, Y., Yamato, H., Takamine, K., Tamaki, H., and Kiyoshi, I. (2010). Analysis of volatile compounds in shochu koji, sake koji, and steamed rice by gas Chromatography-Mass spectrometry. J. Inst. Brew. 116, 49-55. doi: 10.1002/ j.2050-0416.2010.tb00397.x

Yu, H., Dai, X., Yao, G., and Xiao, Z. (2014). Application of gas ChromatographyBased electronic nose for classification of chinese rice wine by wine age. Food Anal. Methods 7, 1489-1497. doi: 10.1007/s12161-013-9778-2

Yu, Y. J. (2014). Organic Acids Analysis and Enhanced Lactic Acid Synthesis of Zhenjiang Aromatic Vinegar. Master's thesis, University of Jiangnan, Jiangsu, IL.

Zhang, X., Tian, X., Ma, L., Feng, B., Liu, Q., Yan, L., et al. (2015). Biodiversity of the symbiotic bacteria associated with toxic marine dinoflagellate Alexandrium tamarense. J. Biosci. Med. 3, 23-28. doi: 10.4236/jbm.2015.36004

Zhao, X. (2016). Fermentation Process of Black Glutinous Rice Wine by Enzyme in Synergy With Microorganisms. Master's thesis, University of Guizhou, Guizhou, IL.

Conflict of Interest: The authors declare that the research was conducted in the absence of any commercial or financial relationships that could be construed as a potential conflict of interest.

Copyright (C) 2020 Jiang, Su, Mu and Mu. This is an open-access article distributed under the terms of the Creative Commons Attribution License (CC BY). The use, distribution or reproduction in other forums is permitted, provided the original author(s) and the copyright owner(s) are credited and that the original publication in this journal is cited, in accordance with accepted academic practice. No use, distribution or reproduction is permitted which does not comply with these terms. 